\title{
GPI-anchored FGF directs cytoneme-mediated bidirectional signaling to self- regulate tissue-specific dispersion
}

\author{
Lijuan Du, Alex Sohr, Sougata Roy* \\ Department of Cell Biology and Molecular Genetics; University of Maryland, College \\ Park, MD 20742
}

*Corresponding author: sougata@umd.edu

Keywords: FGF, bidirectional signaling, GPI anchor, cytonemes, synapse, selforganization, Bnl, cell-adhesion-molecule (CAM), Drosophila

Short Title: Bidirectional FGF-FGFR signaling at cytoneme contacts 


\section{ABSTRACT}

How signaling proteins generate and distribute a multitude of information to shape tissues is critical to understanding morphogenesis. In Drosophila, FGF produced in wing-disc cells regulates the development of the disc-associated air-sacprimordium/ASP. We discovered that FGF signaling is bidirectional and occurs at a distance between ASP and wing-disc cells through physical contacts of filopodia/cytonemes produced by both cells. FGF is GPI-anchored to the source cytoneme membrane, inhibiting its free secretion but facilitating its contact-dependent binding with FGF-receptors/FGFR on ASP cytonemes, thereby producing cytonemecytoneme contacts. Contact-mediated bidirectional FGF-FGFR signaling self-promotes by inducing source and ASP cells to polarize cytonemes toward each other to strengthen inter-cytoneme contacts. Subsequent un-anchoring of FGFR-bound-FGF dissociates contacts and releases FGF target-specifically to ASP cytonemes for paracrine functions. Thus, unlike traditional models of randomly secreted diffusible signals and their one-way directives to recipient cells, GPI-anchored FGF directs both source and recipient cells and self-regulates tissue-specific signaling. 


\section{Introduction}

During morphogenesis, cells coordinate their differentiation, activity, and organization by communicating with conserved families of secreted signals or morphogens ${ }^{1}$. Genetic and molecular characterization of these signaling proteins, including Fibroblast Growth Factor (FGF), Hedgehog (Hh), Wingless (Wg)Wnt, Epidermal Growth Factor (EGF), and Decapentaplegic (Dpp - a BMP homolog), revealed their essential and versatile roles ${ }^{1}$. These signals can disperse across tissues and, upon binding to receptors in recipient cells, activate gene regulatory pathways that specify cell fates, shapes, polarity, and discrete patterns of organization ${ }^{1}$. Strikingly, the same set of signals and their pathways can specify a wide variety of fates and functions in diverse contexts ${ }^{1}$. This raises a question on how these signals might inform cells of their spatiotemporal identity, directionality, activity, and organization to sculpt tissues and how the same pathways might produce variable adaptive tissue-specific information and outcomes.

Traditional models suggest that the signals are readily secreted from producing cells and randomly diffuse through the extracellular space to deliver morphogenetic information to recipient cells. However, advances in live microscopy revealed that during development, signal-exchanging cells extend actin-based signaling filopodia named cytonemes and transmit signals selectively through the sites of cytoneme-cell contacts $^{2-6}$. This neuron-like synaptic signaling through cytonemes and cytoneme-like filopodia are prevalent in diverse vertebrate and invertebrate cells and in various morphogenetic, stem cell niches, and disease contexts ${ }^{2,7-10}$. Essential roles of signaling filopodia in communicating signals, including Hh, Dpp, FGF, EGF, and Wnt, suggest that contact-based signaling is an evolutionarily conserved mechanism ${ }^{3-5,7,8,11-15}$.

A critical feature of cytoneme-mediated signaling is that it can produce diverse recipientspecific patterns of signal dispersion and interpretation ${ }^{6,16}$. For instance, a secreted FGF family morphogen, Branchless (Bnl), is expressed by a group of cells in the Drosophila larval wing imaginal disc, but it forms a long-range dispersion gradient only within the disc-associated air-sac primordium (ASP) that expresses the Bnl receptor Breathless (FGFR/Btl) ${ }^{6,17}$. ASP-specific shapes of Bnl gradients emerge due to the 
graded number of cytonemes along the distal-proximal axis of the tubular ASP that contain Btl and that directly contact Bnl-expressing disc cells to take up $\mathrm{Bnl}^{6}$. On the other hand, varied Bnl signaling levels in different ASP regions activate different target genes that feedback control the formation of Bnl-receiving cytonemes, forming their graded patterns. Consequently, robust recipient-specific shapes of long-range signal and signaling patterns emerge in coordination with ASP growth. These previous results suggested that the initiation of signal dispersion and development of its dynamic and recipient-specific patterns depend on where and when source and recipient cells establish signaling contacts through cytonemes. This led us to the premise that the molecular and cellular processes that produce cytonemes contacts and control signal release specifically through cytoneme contacts are the critical determinants of the spatiotemporal cues for morphogenesis.

In this study, we investigated these mechanisms by examining the inter-organ Bnl dispersion from the wing-disc Bnl-source to the recipient ASP. Like most paracrine signals, Bnl is predicted to be a readily secreted diffusible protein that produces different mitogenic, morphogenic, and motogenic responses in diverse recipient cells, including in stem cells, trachea, neuron/glia, and blood cells, by activating its receptor, Btl ${ }^{14,17-21}$. Contrary to canonical models that depend on random and unidirectional propagation of signals from source to recipient cells, we discovered that $\mathrm{Bnl}$ is anchored to the source cell surface by a glycosylphosphatidylinositol (GPI) moiety. In this form, Bnl acts as a cell adhesion molecule (CAM) that can simultaneously be a receptor and ligand of $\mathrm{BtI} / \mathrm{FGFR}$ and induces bidirectional Btl-Bnl signaling in both source and recipient cells. We further characterized how this CAM-like bidirectional signaling enables the source and recipient cells to collaborate with each other over distance by projecting cytonemes and influencing each other through dynamic inter-cytoneme contacts. We also showed that although the GPI-anchor inhibits random Bnl secretion, it facilitates Bnl release at the inter-cytoneme synaptic contacts. This contact-dependent release is essential for Bnl's long-range tissue-specific dispersion and dose-dependent paracrine signaling. Lipid modification is essential for morphogenetic functions of many signals ${ }^{22-24}$. Therefore, our findings showing how lipidation enables Bnl to program the source and 
recipient cells to reciprocally coordinate with each other by cytonemes and, thereby, self-regulate its tissue-specific dispersion and interpretation provide important new insights into why and how lipidation could facilitate morphogenetic functions for other signals.

\section{Results}

\section{Bidirectional matchmaking of Bnl-sending and Bnl-receiving cytonemes.}

Inter-organ Bnl signaling between the ASP and wing disc is a simple genetic system for an unbiased interpretation of tissue-specific dispersion as $\mathrm{Bnl}$ is produced in the wing disc and travels across a layer of interspersed myoblasts to the overlaying ASP, which expresses its only receptor, Btl (Fig. 1A,B) ${ }^{6}$. Although $B n l$ is a secreted protein, externalized $\mathrm{Bnl}\left(\mathrm{Bnl}^{\mathrm{ex}}\right)$ detected by anti-Bnl immunostaining of non-permeabilized imaginal disc preparations ( $\alpha \mathrm{Bnl}^{\mathrm{ex}}$; Materials and Methods) is not broadly distributed in the myoblasts and extracellular plane of its wing disc source. Instead, Bnl ${ }^{\text {ex }}$ puncta were exclusively localized tissue-specifically only on the basal surface of wing disc source and recipient ASP and ASP cytonemes (Figs. 1A,C). These results are consistent with inter-organ ASP-specific transport of $\mathrm{BnI}^{\mathrm{ex}}$ in a receptor-bound form along the surface of ASP cytonemes (Figs. 1A,C) ${ }^{6}$. Strikingly, compared to the relatively large bnl expression domain, $\mathrm{Bnl}^{\mathrm{ex}}$ puncta on source cells were asymmetrically congregated at the sites where Btl-containing ASP cytonemes established contacts (Fig. 1C).

To examine if the distribution of Bnl in source cells is spatially biased toward the ASP, we examined wing discs that overexpress Bnl:GFP and mCherryCAAX driven by bnlGal4. Although both constructs were expressed under the same driver, only Bnl:GFP puncta were highly enriched in the ASP-proximal source area (Figs. 1D-D"; S1A,A'). Furthermore, wing discs that expressed Bnl:GFP either under endogenous control (bnl:gfp ${ }^{\text {endo }}$ allele $)^{6}$ or under bnl-Gal4, revealed that source cells in the ASP-proximal area extend Bnl:GFP-containing filopodia or cytonemes toward the ASP (Figs. 1D; $\mathrm{S1B})$. These results suggested that the cellular components in source cells responsible for Bnl secretion, display, and/or delivery are polarized toward the ASP. 
Because Bnl-containing filopodia from source cells had not been reported before, we first examined their functions. Source cytonemes were detected in unfixed wing discs that expressed a fluorescent membrane marker (e.g., CD8:GFP or CherryCAAX) either in all of the bnl-expressing cells or in small clones of cells within the Bnl-expressing area. Three-dimensional $X Z Y$ image projections revealed that each of the Bnlexpressing columnar cells proximal to the ASP extended $\sim 2-4$ short $(<15 \mu \mathrm{m})$ cytonemes perpendicularly from their basal surface (Figs. 1F-H; S1C-E; Movie S1; Table S1). The organization of the source cells therefore can be described as polarized for basal Bnl presentation with basal projections extending toward the ASP. This organization is mirrored in the ASP, which, as we previously reported, exhibits polarized Btl synthesis, Bnl reception, and cytoneme orientation toward source cells ${ }^{6}$. Thus, signal-sending and -receiving components polarize to face each other, forming a tissuelevel niche at the ASP:source interface to promote cytoneme-mediated interactions.

Time-lapse imaging of ex vivo cultured wing discs revealed that the ASP and source cytonemes orient toward each other and transiently contact each other's tips (Fig. 1I), bases (Fig. 1J), or shafts (Fig. 1J') as they dynamically extend and retract (Figs. 1I-M; $\mathrm{S} 1 \mathrm{H}-\mathrm{O}$; Table S2; Movies S2a,b). Both cytoneme types had dynamic and repeated cycles of contact association-dissociation, but source cytonemes had a shorter lifetime than ASP cytonemes. Notably, such transient cytoneme:cytoneme contacts at the interface of ASP and source cells are persistent throughout larval development (Fig. $\mathrm{S} 1 \mathrm{H}-\mathrm{M})$. This is consistent with the model of a cytoneme-forming signaling niche at the interface of the ASP and wing disc source.

Based on our previous results on cytoneme-mediated Bnl uptake in the ASP ${ }^{6}, \mathrm{Bnl}$ is likely to be exchanged at the inter-cytoneme contact sites. However, it was technically challenging to capture Bnl exchange via dynamic cytoneme interactions. Therefore, we genetically ablated the source cytonemes and analyzed non-autonomous Bnl dispersion into the ASP. Similar to ASP cytonemes ${ }^{4}$, the formation of source cytonemes could be induced by overexpressing an actin modulator formin, Diaphanous (Dia), and were suppressed by dia knockdown in the source (Fig. 2A-E). Notably, a constitutively active 
form of Dia, Dia $\triangle D A D-G F P$, selectively localized at the ASP-proximal source area and at cytoneme tips, which is consistent with a localized increase in f-actin polymerization activity in the cytoneme-producing source area (Figs. 2C; S1F,G). Ablating source cytonemes in bnl:gfp ${ }^{\text {endo }}$ larvae by dia RNAi expression in the source led to the nonautonomous reduction of Bnl:GFP ${ }^{\text {endo }}$ uptake in the ASP, leading to abnormally stunted ASPs (Fig. 2F-H). Thus, source cytonemes are necessary to selectively deliver Bnl to ASP cytonemes and cells.

Inter-cytoneme $\mathrm{Bnl}$ exchange is consistent with reports that $\mathrm{Hh}$ and $\mathrm{Wg}$ are both sent and received by cytonemes ${ }^{13,25,26}$. However, dynamic interactions of Bnl-exchanging cytonemes that are convergently polarized toward each other might also indicate a possibility of contact-dependent reciprocal guidance of source and recipient cytonemes. To test this possibility, we first ablated source cytonemes by dia RNAi expression and analyzed the effects on ASP cytonemes. The absence of source cytonemes nonautonomously reduced the long, polarized ASP tip cytonemes that make signaling contacts with the source (Fig. 2I-K). Since randomly oriented short ASP cytonemes were unaffected, Bnl-sending cytonemes are required for the formation of only polarized Bnl-receiving cytonemes from the ASP.

To determine if ASP cytonemes also influence source cytoneme polarity, we removed ASP cytonemes by expressing a dominant-negative form of Btl (Btl:DN) in the trachea, as reported previously ${ }^{17}$. Indeed, a complete loss of the ASP and ASP cytonemes led to a corresponding non-autonomous loss of polarized source cytonemes (Fig. 2L). Btl:DN expression occasionally produced partial dominant-negative effects, yielding stunted ASPs with few polarized cytonemes. Strikingly, the appearance of polarized cytonemes in each of these ASPs correlated with the appearance of equivalent numbers of polarized source cytonemes, making stable cytoneme:cytoneme contacts (Fig. 2M-O). These results suggested that the inter-cytoneme contacts induce ASP cells to extend Btl-containing cytonemes toward the source and induce source cells to extend Bnl-containing cytonemes toward the ASP. This suggested that ASP and source cells 
reciprocally feedback control each other by establishing direct contacts, guiding each other's cytoneme formation and polarity and, thereby, self-sustaining their contact sites.

\section{$\mathrm{Bnl}$ is tethered to the source cell surface by a GPI anchor.}

Note that Btl:DN cannot signal due to the lack of its intracellular domain, but can bind to Bnl with its extracellular portion ${ }^{17}$. When we probed for the distribution of externalized $\mathrm{Bnl}$ in the Btl:DN expressing samples (Fig. 2M-O), Bnl ${ }^{\mathrm{ex}}$ was selectively enriched on the source and recipient cytonemes that established stable contacts with each other (Fig. 2P-P"). The enrichment of surface Bnl ${ }^{\mathrm{ex}}$ at the inter-cytoneme contacts suggested a possibility that the cytoneme-mediated Btl-Bnl interaction, which is required for intercellular Bnl exchange, might also bring the source and recipient cytoneme together to form contacts. This model assumed that $\mathrm{Bnl}$ is retained on the source cell surface to act as a cell recognition molecule. How might a secreted protein be retained exclusively on the source cell surface, without being randomly dispersed in the extracellular space?

A mechanism emerged by examining the surface distribution of various chimeric Bnl:GFP constructs expressed in cultured Drosophila S2 cells. Bnl is synthesized as a 770 amino acid protein and is known to be cleaved in the Golgi by Furin1 at residue 164 prior to the externalization of its truncated C-terminal signaling portion (Fig. $3 \mathrm{~A} ;{ }^{27}$ ). Therefore, when S2 cells expressed a chimeric Bnl:HA $\mathrm{GFP}_{3}$ construct with $\mathrm{HA}$ (site 1) and GFP (site 3) flanking the Furin cleavage site, the HA-tag localized to the Golgi and the truncated Bnl:GFP 3 fragment was externalized ${ }^{27}$. These observations led to a hypothesis that a Bnl:GFP ${ }_{3}$ Cherry $_{c}$ construct (Fig. 3A), which has an in-frame C-terminal mCherry fusion, would externalize the truncated C-terminal fragment marked with both GFP and mCherry. Unexpectedly, most membrane-localized Bnl:GFP 3 puncta (detected with $\alpha G F P^{\mathrm{ex}}$ ) lacked mCherry, indicating the possibility of a second intracellular cleavage at the $\mathrm{C}$-terminus of the Bnl protein prior to externalization (Fig. 3B-B"').

Bioinformatic analyses (see Methods) revealed that the $\mathrm{Bnl}$ C-terminal tail has a 20 amino acid hydrophobic segment immediately adjacent to a hydrophilic region (Fig. $\left.3 A, A^{\prime}\right)$, similar to signal sequences of pro-GPI-anchored proteins (pro-GPI-APs). The 
signal sequences of pro-GPI-APs are cleaved and replaced with a GPI moiety in the endoplasmic reticulum (ER) prior to trafficking to the cell surface, where GPI-APs are anchored to the outer leaflet of the plasma membrane ${ }^{28}$. Because the presence of Cterminal tags does not prevent glypiation of pro-GPI-APs ${ }^{29}$, we surmised that Bnl glypiation might explain C-terminal cleavage in Bnl:HA $\mathrm{GFP}_{3} \mathrm{Cherry}_{\mathrm{c}}$ and surface distribution of only Bnl:GFP 3 . To investigate Bnl glypiation, we followed a standard phosphoinositide phospholipase C (PI-PLC) assay (Figs. 3C-E; S2A-H; see Methods). PI-PLC specifically cleaves most GPI moieties between the phosphate and glycerol backbone and sheds GPI-APs from the cell surface. When S2 cells expressed $B n l: G F P_{3}, B n: H A_{1} G F P_{3}$ (henceforth Bnl:GFP), untagged Bnl (detected with $\alpha B n l^{\mathrm{ex}}$ ), and a control GFP-GPI construct ${ }^{30}$, all constructs localized on the cell surface and were shed by PI-PLC treatment (Figs. 3C,C',E; S2A-H). In contrast, PI-PLC did not shed a constitutively active Drosophila EGF construct, cSpitz:GFP (Fig. 3C,C',E), which is tethered to the cell membrane due to palmitoylation ${ }^{24}$.

In silico analyses (see Methods) identified $\mathrm{S}^{741}$ of $\mathrm{Bnl}$ as a probable glypiation site ( $\omega$ site). In S2 cells, Bnl:GFP mutant constructs that either lacked the C-terminal 40 amino acid residues (Bnl:GFP $\Delta$ C), or contained mutated $\omega, \omega+1$, and $\omega+2$ sites (Bnl:GFP- $\omega^{\mathrm{m}}$ ) failed to localize on the producing cell surface, even in the absence of PI-PLC treatment (Figs. 3C-E'; S2G,I). However, when we added the transmembrane domain of the mammalian CD8a protein to the C-terminus of Bnl:GFP $\Delta$ C, the protein (henceforth Bnl:GFP $\Delta$ C-TM) was tethered to the cell surface irrespective of PI-PLC treatment (Figs. 3C-E; S2H,I). Moreover, when the Bnl C-terminal signal sequence was fused to the Cterminus of a secreted sfGFP ${ }^{6}$, the resultant bGFP-GPI construct had the same features of a GPI-AP (Fig. 3C-E). Together, these results identified Bnl as a GPI-AP and characterized Bnl's C-terminal signal sequence needed for GPI modification.

To determine if $\mathrm{Bnl}$ is GPI-anchored on wing disc producing cells, we performed PI-PLC treatment and surface $\alpha \mathrm{Bnl}^{\mathrm{ex}}$ immunostaining on ex vivo cultured wing discs ${ }^{6}$ (Fig. $3 \mathrm{~F}$ R). Native Bnl ${ }^{\mathrm{ex}}$ puncta were concentrated on the disc source cells near ASP cytonemes, but their levels were significantly reduced after PI-PLC treatment (Fig. 3F- 
H). When Bnl and the Bnl:GFP, Bnl:GFP $\Delta C$, and Bnl:GFP $\Delta$ C-TM constructs were overexpressed under bnl-Gal4, PI-PLC treatment significantly reduced levels of $\mathrm{Bnl}^{\mathrm{ex}}$ and Bnl:GFP ${ }^{\text {ex }}$, but not Bnl:GFP $\Delta$ C-TM ${ }^{\text {ex }}$ (Fig. 3I-R). However, Bnl:GFP $\Delta C^{\text {ex }}$ puncta were not concentrated on the source cells even without PI-PLC treatment (Figs. 3N$\left.\mathrm{O}^{\prime}, \mathrm{R}\right)$. They were distributed in the wing disc epithelium surrounding the disc source. This suggested that Bnl:GFP $\Delta C^{\text {ex }}$ is readily secreted into extracellular space. These results confirmed that $\mathrm{Bnl}$ is $\mathrm{GPI}$-anchored to the wing disc source cells.

\section{Bnl's GPI anchor programs tissue-specific Bnl dispersion and signaling.}

To investigate if GPI anchoring on the source surface controls Bnl's tissue-specific dispersion, we imaged GPI-modified (Bnl:GFP) and non-GPI-modified (Bnl:GFP $\Delta$ C and Bnl:GFP $\Delta$ C-TM) constructs expressed from the mCherrryCAAX-marked wing disc Bnl source. Overexpressed Bnl:GFP was localized almost exclusively in wing disc producing cells and target-specifically dispersed into the ASP (Fig. 4A; Movie S3). In contrast, Bnl:GFP mutants that lack a GPI anchor (Bnl:GFP $\Delta$ C and Bnl:GFP $\Delta \mathrm{C}_{168}$ ) were also dispersed around disc source cells, without apparent spatial specificity (Fig. 4C; Movie S4). When Bnl:GFP $\Delta$ C dispersion was tracked in live tissues over a period of time under ex vivo culture conditions (Fig. S4), the signal was randomly spread into the source-surrounding disc area within the same tissue plane but without apparent targeting to the ASP. Target-specificity was regained when Bnl:GFP $\Delta \mathrm{C}$ was TMtethered (Bnl:GFP $\triangle \mathrm{C}$-TM) on the source cell surface. However, the dispersion of $\mathrm{Bnl}$ GFP $\triangle \mathrm{C}$-TM puncta was restricted to only a few ASP cells that were directly juxtaposed to the disc source and these puncta in the ASP were abnormally colocalized with the source cell membrane, indicating a defect in the release from their TM tether, leading to the abnormal uptake (Figs. 4B; S3A-B'; S4; Movie S5). Thus, source surface retention is a critical determinant of tissue-specific inter-organ Bnl dispersion and Bnl's GPI anchoring both facilitates target-specific dispersal and inhibits non-selective secretion. 
To determine if tissue-specific dispersion is needed for Bnl's morphogen-like function, we examined spatial patterns of MAPK signaling induced by GPI-modified and non-GPImodified Bnl:GFP variants. When Bnl:GFP was overexpressed from the wing disc source under bnl-Gal4, all of the ASP cells received Bnl:GFP and induced MAPK (nuclear dpERK localization) signaling (Figs. 4D,K; S3C-E'). As expected, Bnl:GFP $\Delta$ CTM distribution and activity were restricted to only a small number of ASP tip cells (Figs. 4E,K; S3C). Bnl:GFP $\Delta \mathrm{C}$ expression in the source induced ASP overgrowth but a significant number of ASP cells that received Bnl:GFP $\Delta \mathrm{C}$ failed to activate nuclear MAPK signaling (Figs. 4F,K; S3F,G). How ASP growth might be controlled is unclear, but the precise coordination of signal dispersion, signaling, and growth was lost with Bnl:GFP $\Delta C$ expression.

To analyze these signaling anomalies further, we generated ectopic gain-of-function (GOF) clones of Bnl:GFP, Bnl:GFP $\Delta$ C-TM, or Bnl:GFP $\Delta$ C directly within the ASP epithelium. We scored only small-sized clones (2-3 cell diameter) within the ASP stalk or transverse connective (TC) that generally do not receive Bnl or activate MAPK signaling. Ectopic Bnl:GFP GOF clones in the ASP stalk induced dpERK within a uniform distance of 2-3 cells surrounding the clone (Figs. 4H, H',K; S3K). In contrast, Bnl:GFP $\Delta$ C-TM moved from its clonal source to only a few of the juxtaposed cells to induce MAPK signaling in them and to organize them into an ectopic tracheal outgrowth (Figs. 4I,K; S3H,I,K). On the other hand, Bnl:GFP $\Delta \mathrm{C}$ GOF clones had wider signal dispersion than Bnl:GFP, but only a few random signal-receiving cells had dpERK (Figs. 4J,K; S3J,K). Thus, GPI anchoring of Bnl promotes spatiotemporal coordination of Bnl dispersion and interpretation with growth.

$\mathrm{Bnl}$ is also known to chemoattract tracheal branch migration ${ }^{19}$. According to the chemo-gradient model that has been proposed for Bnl's function as an inducer of tracheal branching and migration, extracellular Bnl:GFP $\Delta \mathrm{C}$ would be expected to promote long-range tracheal chemotaxis. To examine this possibility, we ectopically expressed the GPI-modified and non-GPI-modified Bnl:GFP variants in the larval salivary gland, a non-essential, trachea-free organ that normally does not express Bnl 
(Fig. 4L-O) ${ }^{27}$. Although Bnl:GFP and Bnl:GFP $\Delta$ C-TM induced tracheal invasion and branching into the salivary gland, Bnl:GFP $\Delta \mathrm{C}$ did not (Fig. 4L-O). Thus, tracheal branching phenotypes do not correlate with the random presence of extracellular Bnl. On the contrary, increased tracheal invasion/branching on producing cells do correlate with the levels of Bnl available on the surface of source cells. As shown in Figure 4P-S, the amount of Bnl:GFP $\Delta \mathrm{C}$ on the surface of the salivary gland is significantly less than either Bnl:GFP or Bnl:GFP $\triangle \mathrm{C}-\mathrm{TM}$.

Together, these results suggested that Bnl retention on the source surface is the key determinant of Bnl's tissue/context-specific dispersion and interpretation patterns. We proposed that cytoneme-mediated exchange between source and target cells is the mechanism that links source surface Bnl retention with its tissue-specific signaling and that GPI-anchored Bnl is the driver of the cytoneme-mediated signaling.

\section{GPI-anchored Bnl acts as a CAM to drive bidirectional matchmaking of source and ASP cytonemes.}

How does the addition of a GPI moiety enable Bnl to drive cytoneme contact assembly/disassembly and contact-dependent signal release? We tested the idea that GPI-anchored Bnl acts as a cell surface CAM that mediates heterotypic receptorligand-dependent target recognition and bidirectional contact matchmaking of cytonemes. This model also predicts that, like other CAMs ${ }^{31}$, contact-dependent BtlBnl signaling is bidirectional, which would reciprocally activate source and recipient cells to project cytonemes toward each other to form signaling contacts. Finally, binding by Btl present on recipient cytonemes also would release Bnl from its anchor to the source membrane and dissociate cytoneme contacts, thereby restricting $\mathrm{Bnl}$ release only to the target-specific cytoneme contacts.

Results from Btl:DN experiments corroborated with Btl's role as a CAM (Fig. 2L-P"). $\mathrm{Btl}: \mathrm{DN}$ retains the extracellular Bnl-binding domain, and therefore $\mathrm{Btl}: \mathrm{DN}$-containing ASP cytonemes were capable of establishing inter-cytoneme contacts and inducing polarized source cytoneme formation (Fig. 2N-P"). To test if surface-tethered Bnl has 
CAM-like bidirectional activity, we investigated how GPI-modified and non-GPImodified Bnl:GFP variants affect source and ASP cytonemes (Figs. 5A-I; S5A-I). Although Bnl:GFP overexpression abnormally brought a large number of source and recipient cells into close proximity due to the ASP overgrowth, both tissues extended polarized cytonemes toward each other (Figs. 5A-C; S5H,I). Increased extensionretraction dynamics of ASP cytonemes suggested high signaling activity (Movie S6; Table S2). In contrast, overexpression of Bnl:GFP $\Delta \mathrm{C}$ significantly suppressed the formation of long polarized cytonemes in both the source and ASP cells (Figs. 5D-F; $\mathrm{S} 5 \mathrm{~A}, \mathrm{~B}, \mathrm{H}, \mathrm{I})$. Short cytonemes, when detectable, lacked any directional bias and rarely contained Bnl:GFP $\Delta$ C. These results are consistent with the idea that GPI-anchored Bnl induces CAM-like bidirectional contact matchmaking.

Importantly, unlike freely dispersed Bnl:GFP $\Delta$ C, Bnl:GFP $\Delta$ C-TM induced both ASP and source cells to extend large numbers of long polarized cytonemes that were stably adhered to each other via their tips and lateral sides (Figs. 5G-J'; S5C-K; Movies S810). Multiple Bnl:GFP $\Delta$ C-TM puncta were localized at the lateral contact interfaces of the source and recipient cytonemes, indicating an increased number and stability of inter-cytoneme contact sites (Figs. 5G,J-J'; S5E-F; Movies S9-10). The increase in the stability of inter-cytoneme contacts might account for an increase in the intensity of bidirectional responses by Bnl:GFP $\Delta$ C-TM than either Bnl:GFP or WT (native Bnl). Thus, Bnl surface anchoring, irrespective of the nature of the anchor, is sufficient for its CAM-like bidirectional signaling and contact matchmaking.

To visualize heterotypic CAM-like Bnl-Btl interactions at the inter-cytoneme contacts, we took advantage of the relatively stable cytonemes produced by Bnl:GFP $\Delta \mathrm{C}-\mathrm{TM}$.

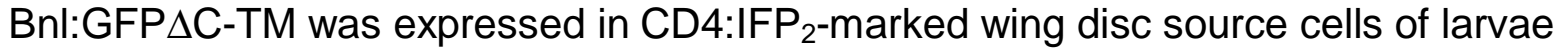
that also harbored a btl:cherry ${ }^{\text {endo }}$ knock-in allele ${ }^{6}$ expressing endogenous Btl:Cherry ${ }^{\text {endo }}$ from the ASP. Btl:Cherry ${ }^{\text {endo }}$ puncta localized in close proximity to $\mathrm{Bnl}$ :GFP $\Delta \mathrm{C}$-TM puncta at multiple sites along the length of the juxtaposed source and recipient cytonemes, mimicking the co-clustered organization of receptors and ligands at the inter-cytoneme junctions (Figs. 6A-B). These results, together with the previous 
evidence (Fig. 1A-C), show that the CAM-like Btl-Bnl-binding through cytoneme contacts is the basis of contact recognition and bidirectional matchmaking.

\section{Bnl's GPI anchor ensures contact-dependent signal release.}

Although Bnl:GFP $\triangle \mathrm{C}-\mathrm{TM}$ acts as a CAM to induce bidirectional responses that manifest in target-specific cytoneme polarity and inter-cytoneme contacts, Bnl:GFP $\Delta \mathrm{C}$ TM-exchanging cytonemes had a significantly longer lifetime than WT or Bnl:GFPexchanging cytonemes (Figs. 6C,D; S5J,K; Movies S8-S11; Table S2). Time-lapse live imaging of source and ASP cells showed that the Bnl:GFP $\Delta$ C-TM-exchanging cytonemes made stable connections that resist signal release, and therefore also resist contact dissociation, leading to cytoneme breakage (Movie S10). Thus, the GPI anchor is required for the release of Btl-bound $\mathrm{Bnl}$ at the contact sites, and this process is likely to dissociate cytoneme contacts. This progressive Btl-Bnl binding, cytoneme contact formation, and contact-dependent Bnl release suggest that the CAM-like function of GPI-anchored Bnl is a prerequisite for its subsequent morphogen-like dispersion. This explains why Bnl:GFP $\Delta$ C-TM exhibits efficient CAM-like cytoneme contact assembly, but is restricted in functional range due to its lack of release, and why Bnl:GFP $\triangle \mathrm{C}$ that lacks CAM activity also fails to induce subsequent morphogenlike organizing functions (Fig. 4D-K; 6E). Only the GPI modification integrates both tissue-specific contact formation and contact-dependent $\mathrm{Bnl}$ release, leading to the diverse context-specific signaling outcomes.

\section{DISCUSSION}

This study uncovered an elegant program of tissue-specific signaling that is encoded by the lipid-modification of an FGF family morphogen, Bnl and orchestrated by cytonememediated contact-dependent communications. On one hand, it showed how cytonemes select a specific target to establish signaling contacts, exchange signals at their contact sites, and dynamically inform cells where they are, what they should do, and when (Fig. $6 \mathrm{E})$. On the other hand, it identified the first lipid-modified FGF and characterized how lipidation enables the signal to program these cytoneme-mediated events and selfcontrol its tissue-specific dispersion and signaling. We showed that this feedback 
program between signaling molecules and cells, controlling each other's location and activity through cytonemes, is critical for dynamic tissue-specific signal dispersion and interpretation in coordination with growth.

According to traditional models, most secreted signals orchestrate morphogenesis by diffusing away from the source and activating gene-regulatory responses in recipient cells. In this one-way directive from a signal to signaling outcomes, signal retention in the source is inhibitory for its subsequent long-range spread and interpretations. In contrast, we discovered that Bnl is GPI anchored to the source cell surface to simultaneously act as a local CAM and a long-range morphogen. GPI modification both inhibits non-selective secretion/diffusion and facilitates its cytoneme-mediated targetspecific long-range spread and bidirectional signaling.

Mechanistically, GPI-anchored Bnl programs three cellular events: cytoneme-mediated target selection, target-specific signal release, and feedback regulations of these processes (Fig. 6E). Our results suggested that GPI-anchored Bnl on the source cell surface and Btl on the receiving cell surface act as heterophilic CAMs. Consequently, when Bnl source and recipient cells extend cytonemes to present $\mathrm{Bnl}$ and $\mathrm{Btl}$ on their surfaces, Btl-Bnl binding leads to the target-specific cytoneme contact formation. Cytoneme contact assembly is a mutual matchmaking process of source and recipient cells. The CAM-like Btl-Bnl binding induces forward signaling in the ASP and retrograde signaling in the source, feedback responses of which promote these cells to extend more Bnl-receiving and Bnl-sending cytonemes toward each other. Our results suggest that by employing these reciprocal cytoneme-promoting feedbacks, source and ASP cells inform each other of their relative locations and directions, thereby establishing a convergently polarized signaling niche to self-sustain contact-mediated interactions.

The purpose of Btl-Bnl-dependent contact recognition is to release the Btl-bound Bnl specifically at cytoneme contact sites. Bnl release not only initiates target-specific Bnl delivery and long-range paracrine functions but also induces cytoneme contact disassembly. Functional attributes of GPI-modified and non-GPI-modified Bnl variants 
suggest that the plasticity of signaling contacts modulates the levels and duration of Bnl signaling. Plasticity of contact is also the key for spatiotemporal adaptability/plasticity in the emerging shapes/patterns. How the local contact-dependent Bnl exchange might self-organize large-scale tissue-specific dispersion/signaling patterns can also be explained from our previous findings on Bnl gradient formation ${ }^{6}$. Thus, the GPI anchor enables Bnl to simultaneously modulate both target-specific cytoneme contact formation and contact-dependent release, thereby linking these two events to be interdependent in time and space via signaling contacts. Here, the molecular, cellular, and tissue level events are interdependent and are integrated by cytoneme contacts.

This mechanism of cytoneme contact formation is reminiscent of CAM-mediated bidirectional pre-synaptic and post-synaptic filopodial matchmaking that organizes Drosophila neuromuscular junctions ${ }^{32}$. Bidirectional transmission of information is the basis for neuronal synaptic assembly, plasticity, and functions, suggesting the conservation of this signaling strategy in both neuronal and non-neuronal cells. A significant consequence of contact-dependent Btl-Bnl bidirectional signaling is that the cause and effects of Bnl signaling can control each other. The same cytoneme contacts that Btl-Bnl binding helps to establish also bring Btl and Bnl molecules together to interact. Thus, not only is the signal exchange cytoneme or contact-dependent, but the cytoneme contacts are also formed signal- or tissue-specifically. Similarly, Bnl and Btl each can act as both a ligand and a receptor for the other. Consequently, contactdependent Btl-Bnl signaling is bidirectional that specifies both source and recipient cells.

These results suggest that the cytoneme-mediated signaling is designed to orchestrate dynamic context-specific self-organization of signal dispersion and interpretation in coordination with tissue growth. Self-organization is an inherent property of living systems. How morphogens might generate self-organized patterns and how signaling patterns might dynamically coordinate with tissue growth are long-standing questions. Contrary to traditional diffusion-based models ${ }^{33}$, our results provide evidence that GPIanchoring/lipidation and source surface retention of a morphogen enables it to program diverse tissue-specific self-organization of cytoneme-mediated signaling. 
Diverse post-translational lipid modifications are critical for the morphogenetic activity of many morphogens, including $\mathrm{Hh}$, Wnt, and EGF/Spi ${ }^{22-24}$. Similar to Bnl, non-lipidated Hh, Spi, and Wnt have unrestricted spread but poor morphogenetic potency, and their engineered TM-tethered forms can induce morphogen-like patterning, despite being limited in the range of dispersion ${ }^{24,34-38}$. Moreover, all signals, including those that are not known to be lipidated (e.g., BMPs and many FGFs), are retained on the cell surface by their interaction with GPI-anchored glypicans, and this process is critical for signal interpretation ${ }^{39-41}$. However, the functional links of these various other signal lipidation and/or retention strategies to cytoneme-mediated bidirectional signaling and/or tissuespecific self-organization of signaling patterns need to be examined in the future.

\section{Acknowledgments}

We thank Drs. N. Andrews and T.B. Kornberg for comments on the manuscript; Drs. T.B. Kornberg and G. O. Barbosa for sharing the design of the culture chamber for live imaging; the Bloomington Stock Center for Drosophila lines; the Developmental Studies Hybridoma Bank for antibodies; Dr. A.E. Beaven for the help in the UMD imaging core facility. Funding: NIH grant R35GM124878 to SR. Author Contributions: A. Sohr discovered GPI-anchoring of Bnl and L. Du discovered its roles; S. Roy supervised the work and designed the project; L. Du and A. Sohr designed and conducted the experiments: S. Roy, L. Du, and A. Sohr wrote the paper. Competing interests: None declared. 


\section{References}

1. Perrimon, N., Pitsouli, C. \& Shilo, B.-Z. Signaling mechanisms controlling cell fate and embryonic patterning. Cold Spring Harbor Perspectives in Biology 4, a005975-a005975 (2012).

2. Kornberg, T. B. \& Roy, S. Cytonemes as specialized signaling filopodia. Development 141, 729-736 (2014).

3. Roy, S., Hsiung, F. \& Kornberg, T. B. Specificity of Drosophila cytonemes for distinct signaling pathways. Science 332, 354-358 (2011).

4. Roy, S., Huang, H., Liu, S. \& Kornberg, T. B. Cytoneme-mediated contactdependent transport of the Drosophila decapentaplegic signaling protein. Science 343, 1244624-1244624 (2014).

5. Bischoff, M. et al. Cytonemes are required for the establishment of a normal Hedgehog morphogen gradient in Drosophila epithelia. Nat Cell Biol 15, 12691281 (2013).

6. Du, L., Sohr, A., Yan, G. \& Roy, S. Feedback regulation of cytoneme-mediated transport shapes a tissue-specific FGF morphogen gradient. (2018). doi:10.7554/eLife.38137

7. Sanders, T. A., Llagostera, E. \& Barna, M. Specialized filopodia direct long-range transport of SHH during vertebrate tissue patterning. Nature 497, 628-632 (2013).

8. Eom, D. S. \& Parichy, D. M. A macrophage relay for long-distance signaling during postembryonic tissue remodeling. Science 355, 1317-1320 (2017).

9. Yamashita, Y. M., Inaba, M. \& Buszczak, M. Specialized Intercellular Communications via Cytonemes and Nanotubes. Annu. Rev. Cell Dev. Biol. 34, 59-84 (2018).

10. Fereres, S., Hatori, R., Hatori, M. \& Kornberg, T. B. Cytoneme-mediated signaling essential for tumorigenesis. PLoS Genet 15, e1008415 (2019).

11. Huang, H. \& Kornberg, T. B. Cells must express components of the planar cell polarity system and extracellular matrix to support cytonemes. Elife 5, 197 (2016).

12. Inaba, M., Buszczak, M. \& Yamashita, Y. M. Nanotubes mediate niche-stem-cell signalling in the Drosophila testis. Nature 523, 329-332 (2015). 
13. Chen, W., Huang, H., Hatori, R. \& Kornberg, T. B. Essential basal cytonemes take up Hedgehog in the Drosophila wing imaginal disc. Development 144, 3134-3144 (2017).

14. Du, L. et al. Unique patterns of organization and migration of FGF-expressing cells during Drosophila morphogenesis. Dev. Biol. 427, 35-48 (2017).

15. Routledge, D. \& Scholpp, S. Mechanisms of intercellular Wnt transport. Development 146, dev176073 (2019).

16. Hatori, R. \& Kornberg, T. B. Hedgehog produced by the Drosophila wing imaginal disc induces distinct responses in three target tissues. Development 147, dev195974 (2020).

17. Sato, M. \& Kornberg, T. B. FGF is an essential mitogen and chemoattractant for the air sacs of the drosophila tracheal system. Developmental Cel/ 3, 195-207 (2002).

18. Sutherland, D., Samakovlis, C. \& Krasnow, M. A. branchless encodes a Drosophila FGF homolog that controls tracheal cell migration and the pattern of branching. Cell 87, 1091-1101 (1996).

19. Ochoa-Espinosa, A. \& Affolter, M. Branching morphogenesis: from cells to organs and back. Cold Spring Harbor Perspectives in Biology 4, a008243-a008243 (2012).

20. Chen, F. \& Krasnow, M. A. Progenitor outgrowth from the niche in Drosophila trachea is guided by FGF from decaying branches. Science 343, 186-189 (2014).

21. Destalminil-Letourneau, M., Morin-Poulard, I., Tian, Y., Vanzo, N. \& Crozatier, M. The vascular niche controls Drosophila hematopoiesis via fibroblast growth factor signaling. Elife 10, 45 (2021).

22. Porter, J. A., Young, K. E. \& Beachy, P. A. Cholesterol modification of hedgehog signaling proteins in animal development. Science 274, 255-259 (1996).

23. Willert, K. et al. Wnt proteins are lipid-modified and can act as stem cell growth factors. Nature 423, 448-452 (2003).

24. Miura, G. I. et al. Palmitoylation of the EGFR ligand Spitz by Rasp increases Spitz activity by restricting its diffusion. Developmental Cell 10, 167-176 (2006). 
25. González-Mendez, L., Seijo-Barandiarán, I. \& Guerrero, I. Cytoneme-mediated cell-cell contacts for Hedgehog reception. Elife 6, 605 (2017).

26. Mattes, B. et al. Wnt/PCP controls spreading of Wnt/ $\beta$-catenin signals by cytonemes in vertebrates. Elife 7, 180 (2018).

27. Sohr, A., Du, L., Wang, R., Lin, L. \& Roy, S. FGF cleavage is required for efficient intracellular sorting and intercellular dispersal. J Cell Biol (2019). doi:10.1083/jcb.201810138

28. Saha, S., Anilkumar, A. A. \& Mayor, S. GPI-anchored protein organization and dynamics at the cell surface. J. Lipid Res. 57, 159-175 (2016).

29. Caras, I. W. An internally positioned signal can direct attachment of a glycophospholipid membrane anchor. J Cell Biol 113, 77-85 (1991).

30. Greco, V., Hannus, M. \& Eaton, S. Argosomes: a potential vehicle for the spread of morphogens through epithelia. Cell 106, 633-645 (2001).

31. Hynes, R. O. Integrins: bidirectional, allosteric signaling machines. Cell 110, 673687 (2002).

32. Kohsaka, H. \& Nose, A. Target recognition at the tips of postsynaptic filopodia: accumulation and function of Capricious. Development 136, 1127-1135 (2009).

33. Green, J. B. A. \& Sharpe, J. Positional information and reaction-diffusion: two big ideas in developmental biology combine. Development 142, 1203-1211 (2015).

34. Taylor, F. R. et al. Enhanced potency of human Sonic hedgehog by hydrophobic modification. Biochemistry 40, 4359-4371 (2001).

35. Gallet, A., Ruel, L., Staccini-Lavenant, L. \& Thérond, P. P. Cholesterol modification is necessary for controlled planar long-range activity of Hedgehog in Drosophila epithelia. Development 133, 407-418 (2006).

36. Callejo, A., Torroja, C., Quijada, L. \& Guerrero, I. Hedgehog lipid modifications are required for Hedgehog stabilization in the extracellular matrix. Development 133, 471-483 (2006).

37. Speer, K. F. et al. Non-acylated Wnts Can Promote Signaling. CellReports 26, 875-883.e5 (2019).

38. Alexandre, C., Baena-Lopez, A. \& Vincent, J.-P. Patterning and growth control by membrane-tethered Wingless. Nature 505, 180-185 (2014). 
39. Yan, D. \& Lin, X. Shaping morphogen gradients by proteoglycans. Cold Spring Harbor Perspectives in Biology 1, a002493-a002493 (2009).

40. Dejima, K., Kanai, M. I., Akiyama, T., Levings, D. C. \& Nakato, H. Novel contactdependent bone morphogenetic protein (BMP) signaling mediated by heparan sulfate proteoglycans. J. Biol. Chem. 286, 17103-17111 (2011).

41. Balasubramanian, R. \& Zhang, X. Mechanisms of FGF gradient formation during embryogenesis. Semin. Cell Dev. Biol. 53, 94-100 (2016).

42. Cabernard, C. \& Affolter, M. Distinct roles for two receptor tyrosine kinases in epithelial branching morphogenesis in Drosophila. Developmental Cell 9, 831842 (2005).

43. Hooper, N. M. Determination of glycosyl-phosphatidylinositol membrane protein anchorage. Proteomics 1, 748-755 (2001).

44. Dye, N. A. et al. Cell dynamics underlying oriented growth of the Drosophila wing imaginal disc. Development 144, 4406-4421 (2017).

45. Sohr, A., Du, L. \& Roy, S. Ex vivo Drosophila Wing Imaginal Disc Culture and Furin Inhibitor Assay. Bio-protocol 9, e3336 (2019).

\section{Figure Legends}

Fig. 1. Bnl source and recipient cells extend cytonemes to contact each other. (A) Drawing depicting organization of the ASP, wing disc, myoblasts, and Btl-containing ASP cytonemes that directly receive Bnl from the disc bn/ source. (B) Polarized ASP cytonemes (green; arrow) establish contacts with the wing disc bn/ source (red). (C) Externalized $\mathrm{Bnl}^{\mathrm{ex}}\left(\mathrm{red}, \alpha \mathrm{Bnl}^{\mathrm{ex}}\right.$ ) is asymmetrically enriched on the source (dashed lined) and Btl:GFP-containing ASP cytoneme contact sites (arrow). (D-D") The wing disc bnl source (red) co-expressing Bnl:GFP and mCherryCAAX (bnl-Gal4 X UAS-Bnl:GFP, UAS-mCherryCAAX); arrowhead, ASP-proximal area; arrow, Bnl:GFP containing cytonemes; dashed line, ASP; D", Bnl:GFP intensity profile within the boxed source area in the direction of the arrow in D' (see Fig. S1A-B). (E,F) 3D-rendered XZY views 
of the nlsGFP-marked ASP and mCherryCAAX-marked source cytonemes (arrow). (G) 3D-rendered views of two CD8:GFP-expressing clones within the $\mathrm{bn} /$ source area; arrow, cytonemes (see Fig. S1D,E; Table S1). (H) Violin plot showing the source cytoneme length distribution. (I-K) Dynamic contact-based interactions of source (red) and ASP (green) cytonemes; K, illustration of the results; arrowhead, contact sites. (L,M) Plots comparing ASP and source cytoneme dynamics (see Material and Methods; Fig. S1N,O; Table S2). All except C, live imaging. Scale bars, $20 \mu \mathrm{m} ; 5 \mu \mathrm{m}(\mathrm{G})$.

\section{Fig. 2. Bidirectional contact matchmaking of $\mathrm{Bnl}$ sending and receiving} cytonemes. (A-E) Autonomous effects of Dia:GFP, Dia $\triangle \mathrm{DAD}: \mathrm{GFP}$ and diaRNAi expression on cytonemes in mCherryAAX-marked source cells; all panels, 3D-rendered $X Z Y$ views; E, graph showing source cytoneme numbers under indicated conditions; $p<$ 0.01 for WT $(n=6)$ vs Dia $(n=6)$ or Dia $\triangle \mathrm{DAD}(\mathrm{n}=8)$ or dia-i $(\mathrm{n}=6)$; (UASmCherryCAAX;bnl-Gal4 X w' for control, or UAS-"X"). (F-H) Bnl:GFP endo uptake in the ASP (dashed line) in control (bnl:gfp ${ }^{\text {endo }} \mathrm{X}$ bnl-Gal4) and source cytoneme-depleted conditions (UAS-dia-i, bnl:gfp ${ }^{\text {endo }} \mathrm{X}$ bnl-Gal4); $\mathrm{H}$, violin plot showing levels of Bnl:GFP endo uptake in the ASP under indicated conditions; $\mathrm{p}<0.0001, \mathrm{n}=7\left(w^{-}\right)$and 17 (dia-I); red, phalloidin-Alexa-647. (I-K) Non-autonomous effects of source cytonemedepletion on ASP cytonemes (CD2:GFP-marked); K, Plots comparing ASP cytoneme numbers under indicated conditions, $\mathrm{p}<0.0001$ for cytonemes $>15 \mu \mathrm{m}, \mathrm{n}=6$ ASPs (control), 14 ASPs (dia-l); dashed arrow, ASP tip cytonemes. (L-O) Tracheal BtI:DN expression that removed ASP cytonemes also non-autonomously removed source cytonemes (red); O, graph showing correlation of source and ASP cytoneme numbers under partial Btl:DN conditions. (P-P") Bnl ${ }^{\mathrm{ex}}$ (blue, $\alpha B \mathrm{Bl}^{\mathrm{ex}}$ ) localization at inter-cytoneme contacts in samples with partial Btl:DN phenotypes; $\mathrm{P}^{\prime}$, split $\alpha B \mathrm{Bl}^{\mathrm{ex}}$ channel; P", zoomed-in part of P. (F-N) dashed arrow, non-autonomous effects of the indicated genetic manipulation (arrow); arrowheads, inter-cytoneme contacts. All panels except F,G, P-P”, live imaging. Scale bars, $20 \mu \mathrm{m}$. 
Fig. 3. Bnl is GPI anchored to the source cell surface. (A,A') Schematic map of the Bnl protein showing FGF domain, secreted signaling portion (left-right arrow), signal peptide (SP), signal sequence (SS), and sites for: furin cleavage (arrow), HA-tag (site \#1), GFP-tag (site \# 3), mCherry-tag, hydrophobicity plot ( $\left.A^{\prime}\right)$, and numbers, amino acid residues. (B-E') S2 cells co-transfected with actin-Gal4 and UAS-"X" constructs and surface immuno-probed in non-permeabilized condition with either GFP or Bnl antibody, as indicated. (B-B',') In S2 cells, Bnl:HA GFP $_{3}$ Cherry $_{\mathrm{c}}$ (arrowheads) is cleaved intracellularly, and Bnl:GFP ${ }_{3}{ }^{\text {ex }}$ portion (arrow; blue) is surface localized; B'-B'”, split channels. (C-E) Surface localization (red) of various constructs under pre-PIPLC and post-PIPLC conditions; CD8:GFP was co-expressed for untagged Bnl as an internal control; D, schematic maps of different Bnl constructs; E, E', Graphs comparing the ratio of surface (red) to total protein (GFP) expressed for indicated constructs and conditions (also see Fig. S2); ${ }^{* *}, \mathrm{p}<0.001, \mathrm{n}=15$ cells. (F-K) Surface $B \mathrm{l}^{\mathrm{ex}}$ (red) levels on the wing disc $b n /$ source (arrows) before and after PIPLC treatment; F-H, native Bnl in w control larvae; I-K, overexpressed Bnl under bnl-Gal4. (L-R) Source surface levels (red) of $B n l: G F P, B n l: G F P \Delta C$ and Bnl:GFP $\Delta$ C-TM on wing discs when expressed under bnlGal4 (control) before and after PIPLC; R, graph comparing the fraction of source surface levels (red, $\alpha G F P^{\mathrm{ex}}$ ) to the total expression levels (green) of different variants; $\mathrm{n}=5$ for each; only merged and red channels shown; arrows, source area; *, sourcesurrounding disc area; dashed line, ASP; Scale bars, $30 \mu \mathrm{m} ; 10 \mu \mathrm{m}$ (B-C').

Fig. 4. GPI anchor ensures tissue-specific Bnl dispersion and interpretation. (A-C) Dispersion patterns of Bnl:GFP, Bnl:GFP $\Delta$ C-TM (TM), or Bnl:GFP $\Delta \mathrm{C}(\Delta \mathrm{C})$ from the wing discs bnl source (UAS-mCherryCAAX; bnl-Gal4 X UAS-"X"); $\mathrm{B}$, arrowhead, abnormal source membrane colocalized Bnl:GFP $\Delta$ C-TM puncta; insets, zoomed-in ROI (box); dashed line, ASP; *, source-surrounding disc area; $\alpha$ Discs large (Dlg), cell outlines. (D-K) Dispersion and signaling (dpERK, red) patterns of Bnl:GFP, TM, and $\Delta \mathrm{C}$ when expressed from either the wing disc bnl source (D-F; bnl-Gal4 $\underline{x}$ UAS-"X") or ectopic GOF clones within the ASP (H-J; hsFlp; btlenh $>y+>$ Gal4, btlenh-mRFPmoe $\underline{x}$ UAS-" $X "$ "); H-J insets, clone positions and signaling patterns (also see Fig. S3H-K); D-J; arrow and arrowhead, signal-recipient cells with and without nuclear dpERK, 
respectively; G, average intensity plots comparing Bnl:GFP, TM, and $\Delta \mathrm{C}$ distribution along the ASP D-P axis; K, violin plots comparing the percentage of signal-receiving ASP cells with nuclear dpERK from overexpression (OE) and clonal expression; $p<$ 0.01: $\Delta C(n=16)$ vs either Bnl:GFP $(n=17)$ or TM $(n=13)$ under OE; see Fig. S3K for p values for clonal assays. (L-O) Levels of tracheal branch invasion (arrows) on larval salivary glands expressing either Bnl:GFP, TM, or $\Delta \mathrm{C}$ (bnl-Gal4 X UAS-X); L-N, brightfield, 10X magnification; $\mathrm{O}$, Sholl analyses graph comparing frequency of terminal branching. (P-S) Fraction of Bnl:GFP, TM, and $\Delta \mathrm{C}$ displayed on the basal surface (red, $\alpha \mathrm{GFP}^{\mathrm{ex}}$ ) when expressed from salivary glands; $\mathrm{S}$, graph comparing the fraction of surface displayed signals (arrowhead); arrow, cell junctions; $p<0.05$ : Bnl:GFP ( $n=17$ ) vs TM ( $n=26)$, $p<0.01: \Delta$ C (29) vs either Bnl:GFP or TM. Scale bars, $30 \mu \mathrm{m} ; 100 \mu \mathrm{m}$ $(\mathrm{L}-\mathrm{N}) ; 20 \mu \mathrm{m}\left(\mathrm{P}-\mathrm{R}^{\prime}\right)$.

\section{Fig. 5. GPI-anchored Bnl acts as a CAM to drive bidirectional cytoneme contact} matchmaking. (A-I) Effect on the reciprocal polarity and numbers of ASP and source cytonemes (arrows) when either Bnl:GFP, Bnl:GFP $\Delta$ C, or Bnl:GFP $\Delta$ C-TM were expressed from the disc source (also see Fig.S5A-G); A,D,G, extended Z- projection, both the ASP (red) and source (blue) are genetically marked; $A$, inset, ROI (box) in green and blue channels; B,E,H, 3D-rendered views, only source membrane marked (red); dashed lines, ASP; B', only red channel of B; D,E, dashed arrows, randomly oriented short cytonemes devoid of signal localization; C,F,I, R-plots comparing numbers, length, and polarity of ASP and source cytonemes (also see Fig. S5H,I). (J,J') 3D projection of cytonemes from the ASP $(b t />C h e r r y C A A X)$ and the Bnl:GFP $\Delta \mathrm{C}-\mathrm{TM}$ expressing wing disc $b n /$ source (blue, $b n l>C D 4: I F P 2)$; J', orthogonal views showing a Bnl:GFP $\Delta$ C-TM puncta (arrow) at an inter-cytoneme junction. All panels, live imaging; dashed line, ASP outline. Scale bars, $20 \mu \mathrm{m}$.

Fig. 6. Btl-Bnl-interactions mediate cytoneme contact assembly and plasticity. (AB) 3D-projected images of cytonemes from the Bnl:GFP $\Delta$ C-TM-expressing wing disc 
bnl source (blue, bnl>CD4:IFP2) in btl:cherry ${ }^{\text {endo }}$ knock-in larvae; Co-clustering of $\mathrm{Btl}:$ Cherry ${ }^{\text {endo }}$ puncta (red) from the ASP and Bnl:GFP $\Delta \mathrm{C}$-TM puncta (arrows) from the source at the inter-cytoneme contact sites. (C,D) Violin plots showing the maximum extension and lifetime $(C)$ and average extension and retraction velocity (D) of ASP (recipient) and source cytonemes while source cells expressed Bnl:GFP $\Delta$ C-TM (also see Table S2 for comparison and statistical significance). All panels, live imaging. (E) Proposed model showing how GPI-anchored Bnl ensures tissue-specific cytoneme contact matchmaking, contact-dependent exchange, and bidirectional signaling feedback to self-sustain signaling sites. Scale bars, $20 \mu \mathrm{m}$. 


\section{Figure 2}

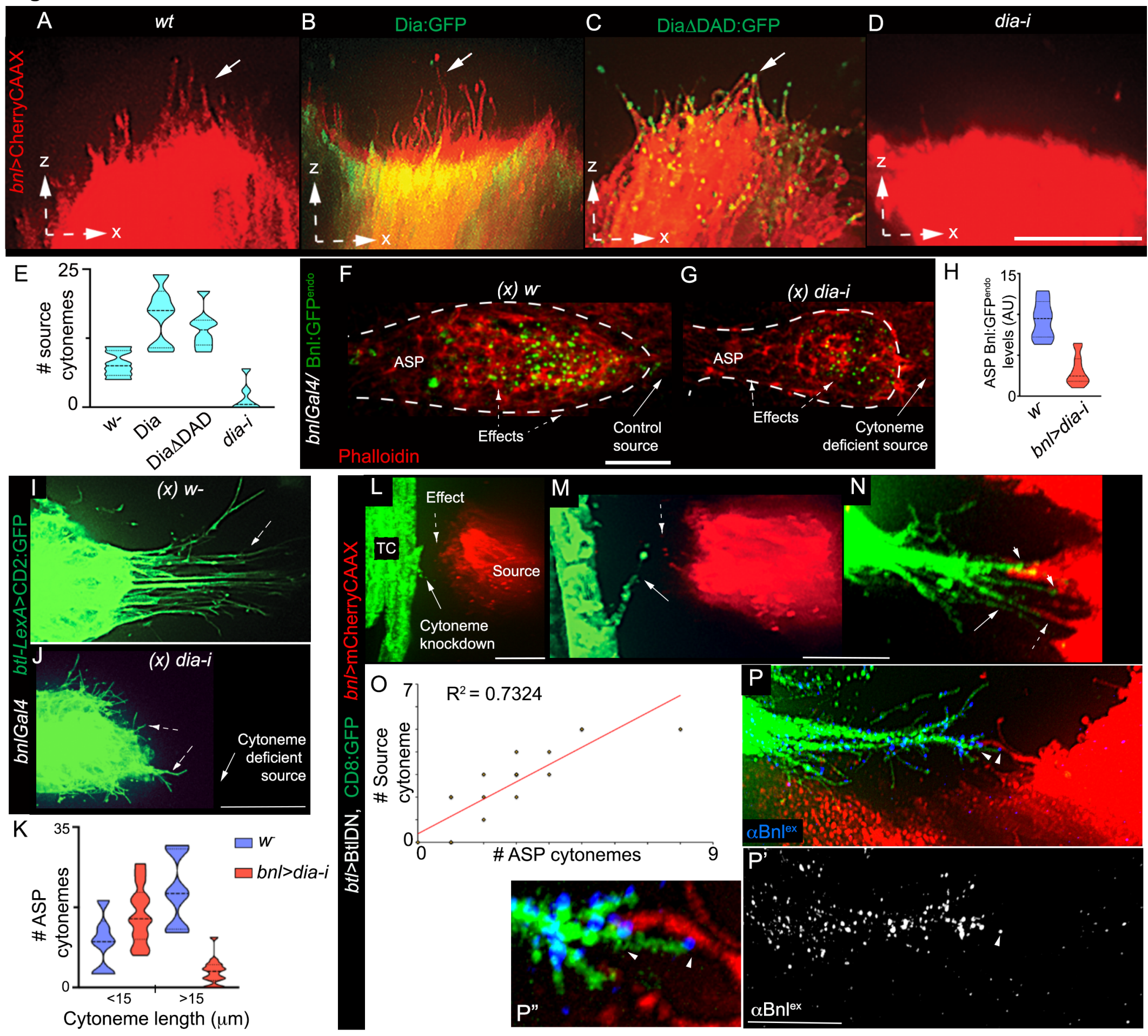




\section{Figure 3}
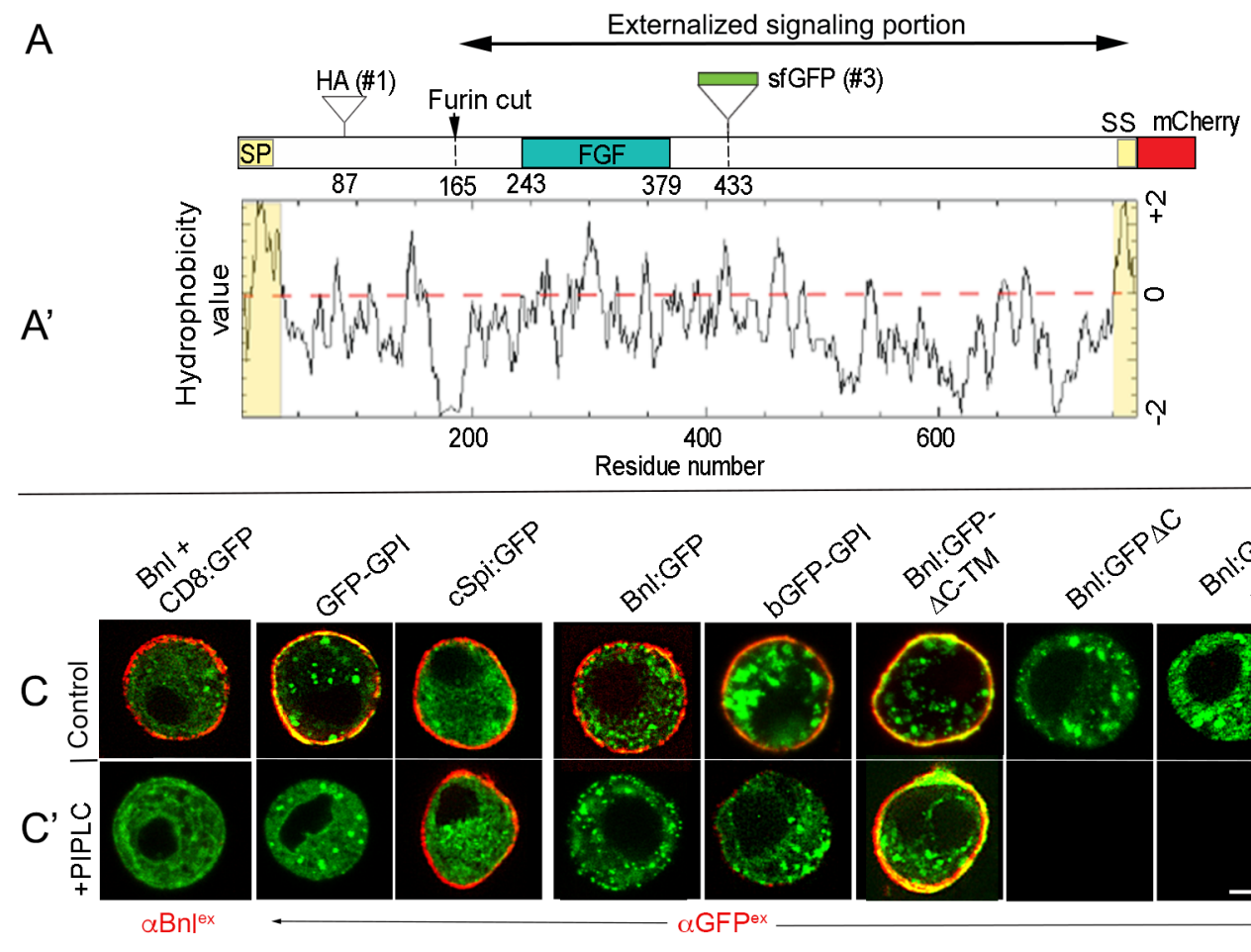

E

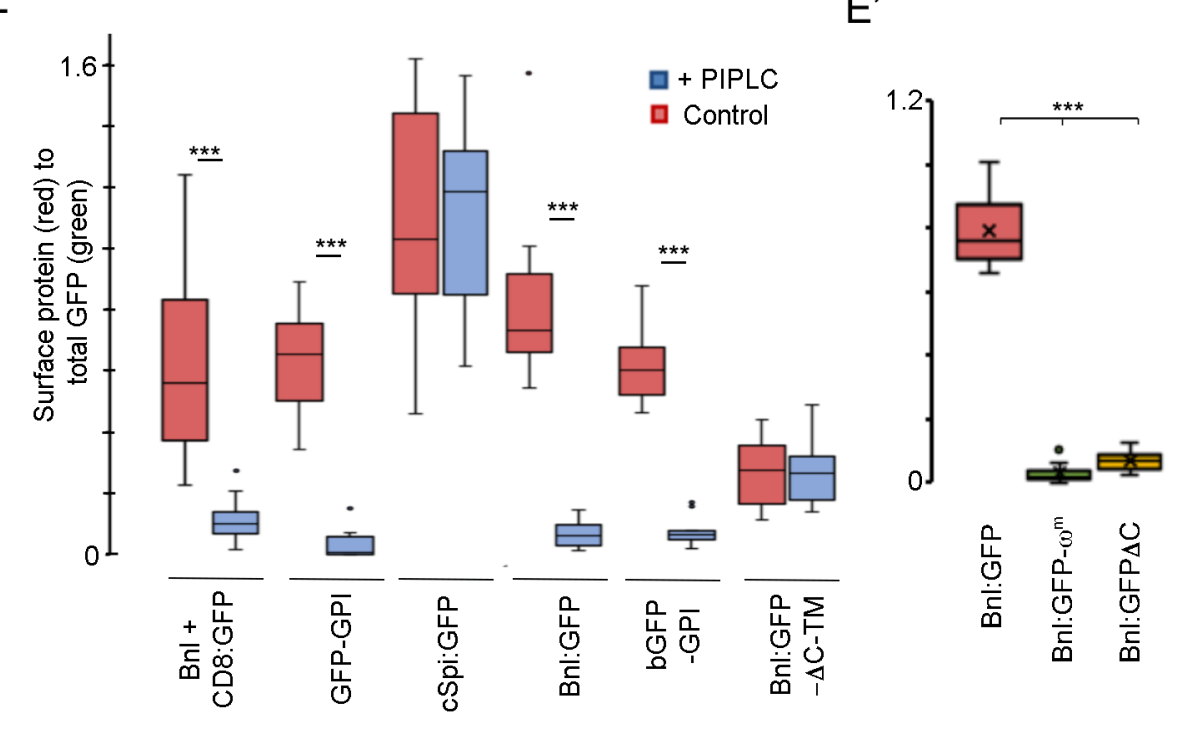

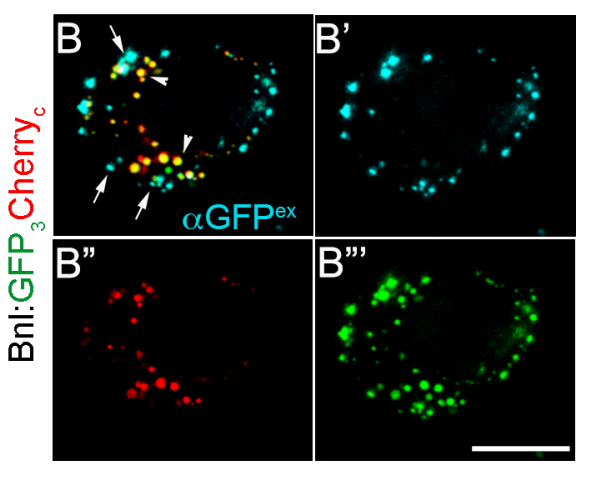
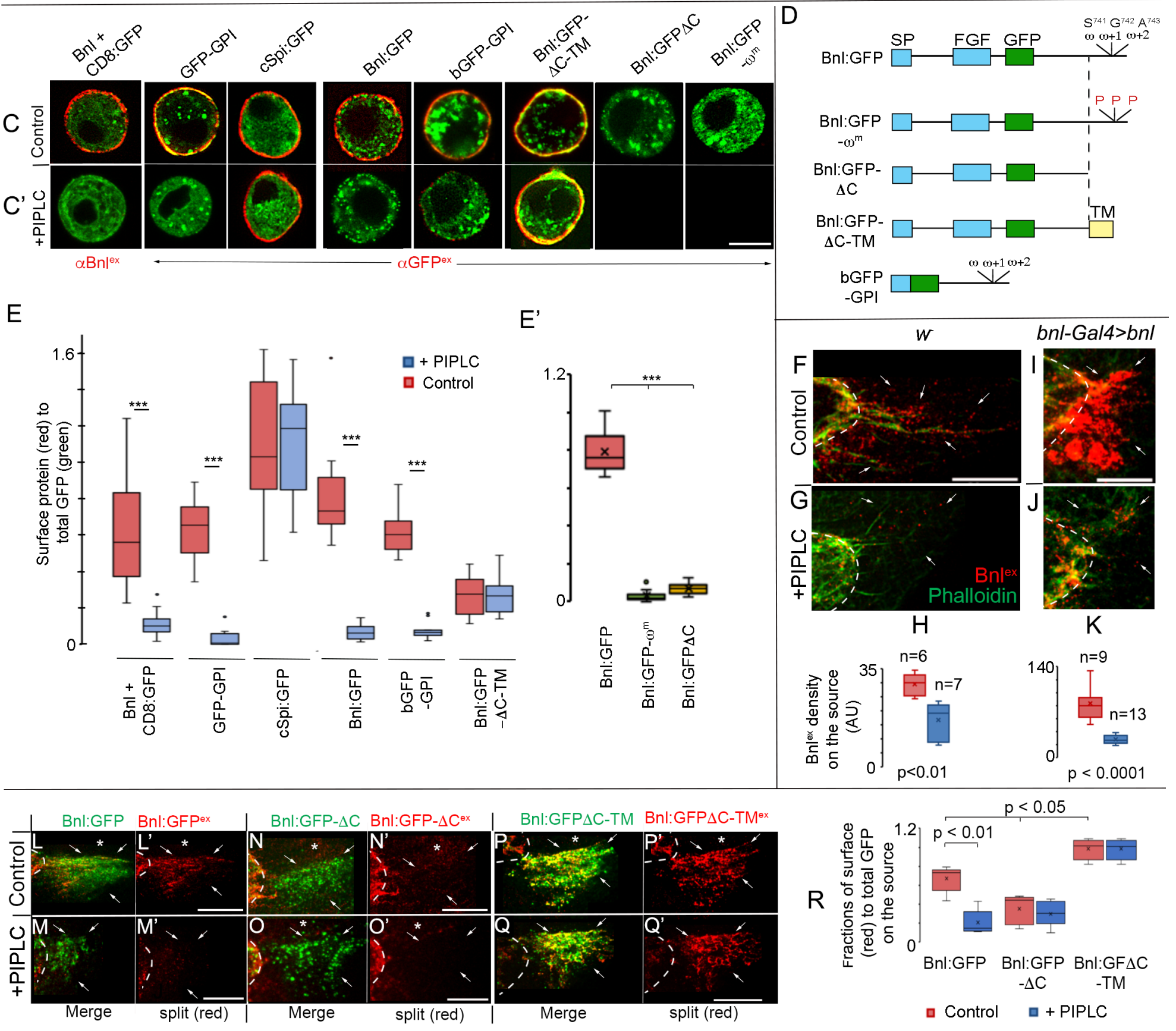


\section{Figure 5}

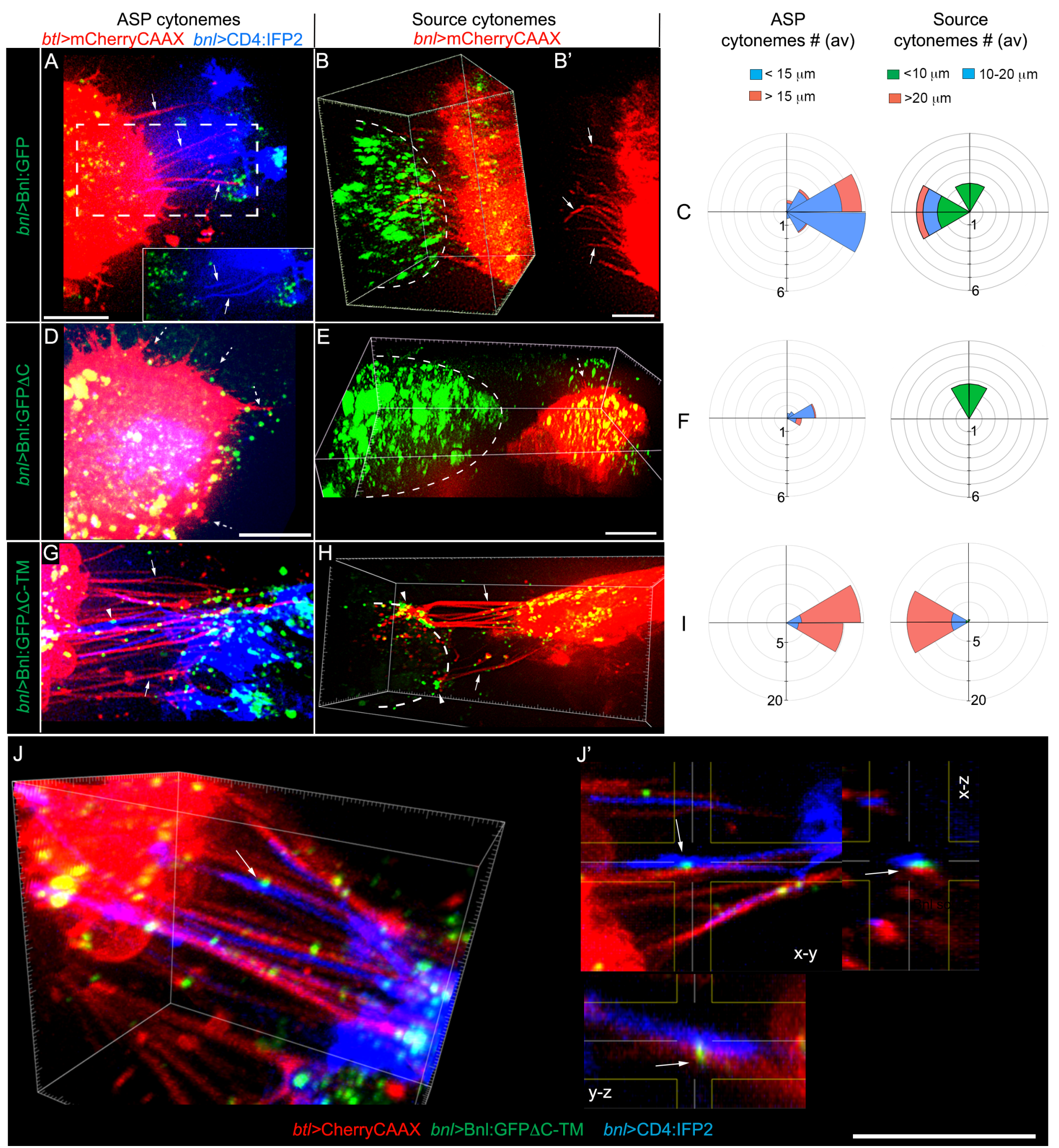




\section{Figure 6}

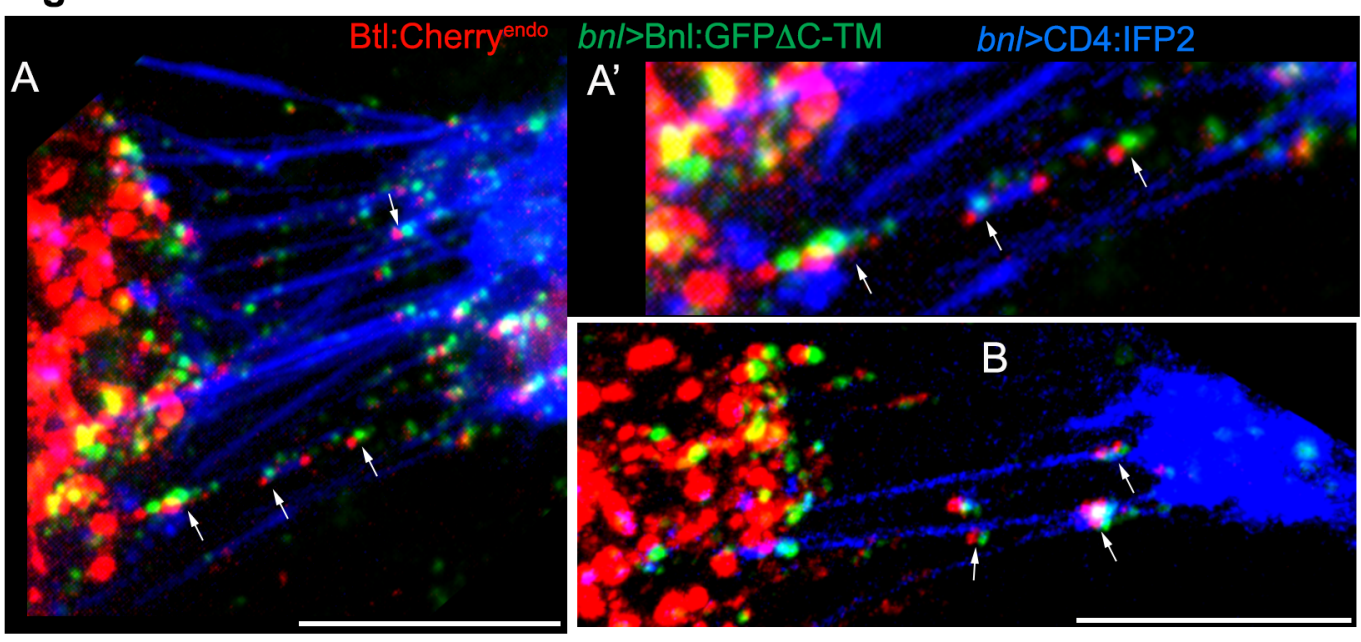

E

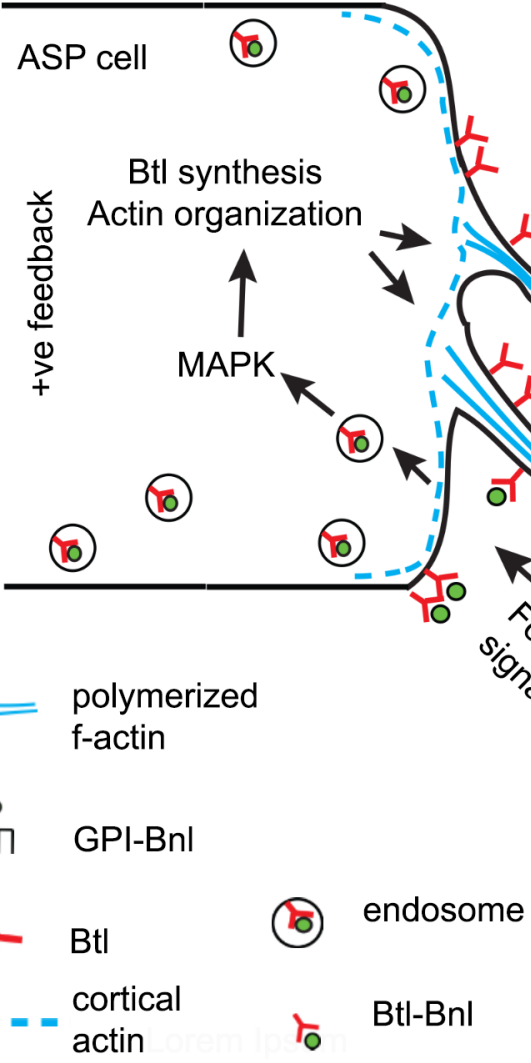

C

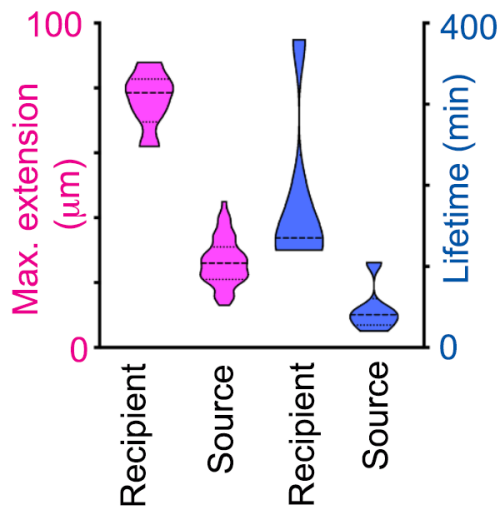

D

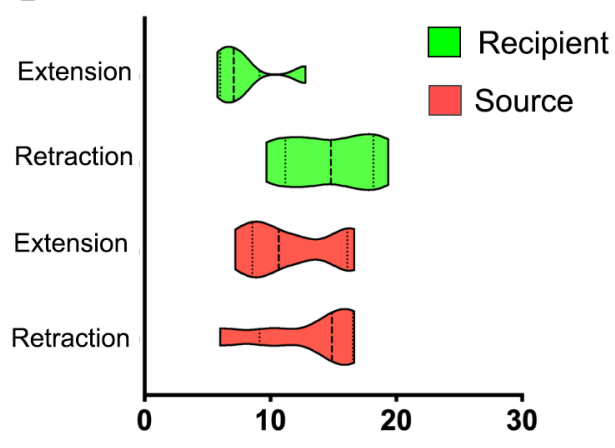

Average velocity $(\mathrm{nm} / \mathrm{s})$ 